\title{
Efficient Removal of Persistent Organic Pollutants (POPs) from Waters and Wastewaters by the Use of lonizing Radiation
}

\author{
Marek Trojanowicz \\ Department of Chemistry, University of Warsaw \\ Institute of Nuclear Chemistry and Technology \\ Warsaw, Poland \\ trojan@chem.uw.edu.pl
}

The necessity of search for a new more efficient methods of removal of anthropogenic pollutants from waters and wastewaters results from a widely documented insufficient efficiency of conventional and routinely used methods of treatment. This concerns first of all the residues of human and veterinary pharmaceuticals, and also numerous groups of industrial and agricultural pollutants of particular resistance, which are considered as Persistent Organic Pollutants (POPs). They are already quite large group of chemicals indicated by Stockholm Convention from 2001, with numerous later novelizations. Their list includes compounds relating to their persistence, bioaccumulation, and potential for long-range environmental transport [1].

One of the most important directions towards search for new treatment processes for decomposition of those pollutants is a development on new treatment methods based on reactions with generated in-situ strongly reactive free radicals, described as Advanced Oxidation Processes (AOPs). For this purpose a different processes are investigated, including photochemical ones, photocatalytic with increasing applications of nanotechnology achievements, ozonation, sonolysis, and electrochemical oxidation. Most commonly for this purpose generation of strongly oxidizing hydroxyl radical $\bullet \mathrm{OH}^{\circ}$ employed.

Especially efficient way of production of $\bullet \mathrm{OH}$ radicals is the irradiation of treated aqueous solutions (containing target pollutants) with gamma-rays from radioisotope sources, e.g. 60Co or with beam of accelerated electrons (EB) from accelerators. A unique feature of this process comparing to other AOPs is simultaneous production as result of water radiolysis a similar amount of hydrated electrons, creates the possibility of conducting simultaneously oxidation and reduction processes, depending on reactivity of given pollutant(s) present in irradiated media.

Those processes are being developed since 1950s and they were already applied in decomposition of numerous groups of pollutants in laboratory scale, pilot plants and also technological installations [2]. Among different POPs those Advanced Oxidation/Reduction Processes were already employed for efficient decomposition of numerous pesticides such as DDT, hexachlorobenzene, lindane or endrin. They were also employed by numerous authors for decomposition of polychlorinated biphenyls (PCBs). In our studies the efficient decomposition of perfluorinated surfactants, including perfluorooctane sulfonate (PFOS) officially regarded as POP, was obtained by hydrated electrons. Besides abovementioned efficiency and possibility of conducting simultaneously oxidation and reduction processes, the application of EB irradiation provides much shorter time of carrying on the decomposition process, comparing to all other AOPs [3].

\section{References}

[1] D. Downie, J. Templeton, "Persistent Organic Pollutants" in The Routledge Handbook of Global Environmental Politics. New York: Routledge, 2013.

[2] M. Trojanowicz, K. Bobrowski, T. Szreder, A. Bojanowska-Czajka, Gamma-ray, "X-ray and electron beam processes as an Advanced Oxidation Processes", in Advanced Oxidation Processes for Wastewater Treatment, S. Ameta, R. Ameta, Eds. Elsevier, 2018.

[3] M. Trojanowicz, A. Bojanowska-Czajka, A. Capodaglio, "Can radiation chemistry supply a highly efficient, economical process alternative for organics removal from water and wastewater?," Environ. Sci. Poll. Res., vol. 24, pp. 20187-20208, 2017. 